\title{
El Dr. Seuss en la encrucijada de la forma y el contenido en castellano
}

\section{Dr. Seuss at the Crossroads of Form and Content in Spanish}

\author{
Guillermo BADENES y Josefina COISSON \\ FACUltad de Lenguas, Universidad Nacional de CóRdoba \\ Argentina
}

\begin{abstract}
Resumen
El dilema de preservar la forma o el contenido ha sido discutido in extenso en los estudios traductológicos durante gran parte del siglo XX. Hayes (1975) confiaba en el sentido común del traductor al momento de encarar el arte de la traducción literaria. Por su parte, con un enfoque más descriptivo, Ben-Ari (1992) y Shavit (1992) estudiaron las características específicas de la literatura infantil traducida del alemán al hebreo revisando variables históricas y entendiendo la fuerza canónica de ciertos libros dentro del catálogo del sistema estudiado. En inglés, como en castellano, la literatura infantil carece en cierta medida de importancia canónica. Sin embargo, existen ejemplos específicos (tal el caso de las obras de grandes autores ingleses del siglo XIX como Lewis Carroll, Stevenson, o C. S. Lewis) que lograron adquirir estatus canónico. Como el epítome de la escritura periférica, la traducción (en este caso la traducción de literatura infantil) deviene una práctica periférica de una literatura periférica. Si consideramos que en el polisistema las obras tienden a ir y venir de posiciones centrales a posiciones periféricas y viceversa, podemos concluir que es importante abrir espacios a culturas alternativas, y que la traducción puede tener un papel preponderante en este proceso. Este trabajo analiza las estrategias utilizadas por tres traductores diferentes de tres obras de la serie de Dr. Seuss. Nuestros hallazgos persiguen hacer foco en las ideas de Hayes, Ben-Ari y Shavit centrados en la literatura para niños.
\end{abstract}

Palabras clave: literatura infantil, Dr. Seuss, polisistemas, traducción

\begin{abstract}
The form-content dilemma was extensively discussed by authors throughout the better part of the 20th century. Hayes (1975) relied on the translator's common sense when approaching the art of literary translation. Taking a more descriptive approach, Ben-Ari (1992) and Shavit (1992) discussed specific features of literature in translation from German into Hebrew revising historical variables and
\end{abstract}


understanding the canonical power of certain children's books within the catalogue of the system under study. In English, as in Spanish, children's literature lacks an important canonical pull. Nonetheless, there are certain specific examples such as the work of the great English authors of the 19th century, Lewis Carroll, Stevenson, or CS Lewis, which not only have become greatly influential in the English language but also in other languages, as is the case of Spanish. As the epitome of peripheral literary writing, translation - in this case the translation of children's textualities - becomes a peripheral practice of a peripheral literature. If we consider that within the polysystem literary works tend to flux from central to peripheral positions and vice versa, we may conclude that it is important to open spaces for alternative cultures, a process that may rely heavily on translation. This paper aims at shedding light upon the strategies used by three different translators of three books belonging to the Dr. Seuss series. Our findings may give focus to Hayes', Ben-Ari's and Shavit's ideas centering on children's literature.

Keywords: children's literature, Dr. Seuss, polysystems, translation

\section{Había una vez...}

El siglo Xx inauguró el periodo científico de la Traductología y también su dependencia de la Lingüística y la Filología, la instauración de un enfoque prescriptivo, la profundización del objeto de estudio, un método y la certeza de un campo de investigación. Se podría sugerir que el auge contemporáneo de la Traductología surgió durante la Segunda Guerra Mundial, cuando las potencias en conflicto buscaban dilucidar los mensajes secretos enemigos a los que accedían los países en guerra. De este modo, la Escuela de Traductores de Moscú se fundó en 1930 y, en la Base Naval de Presidio, en California, se estableció unos años más tarde una división de estudios lingüísticos para la traducción. Sin dudas, este trabajo subrepticio, oculto e ignoto de espías no podría nunca contribuir a pensar en la visibilidad de la traducción. Sin embargo, si bien fue la guerra la que potenció el interés por el estudio de la traducción, fue la paz (el interés por fomentar la concordia entre los pueblos) y la necesidad por formar a los profesionales que integrarían las nuevas organizaciones internacionales, las que dieron origen a las primeras escuelas de traducción donde se comenzó a estudiar la Traductología, de la mano de la Lingüística, la Psicología, la Filosofía y otras disciplinas: "Tras siglos de un interés fortuito y esporádico de parte de un puñado de autores, filólogos y académicos literarios, más algún que otro teólogo o lingüista idiosincrático, la traducción recibió un interés marcado y constante de parte de la academia en años recientes, con la Segunda Guerra Mundial como momento bisagra" (Holmes, 2000: 173). ${ }^{1}$ Tal vez haya sido el hecho de desarrollarse en ámbitos universitarios lo que contribuyó al

\footnotetext{
${ }^{1}$ Traducción de los autores; en adelante todas serán traducciones de los autores, a menos que se especifique lo contrario.
} 
diálogo interdisciplinario de la traducción. Si consideramos que en algunas universidades la Traductología, o los Estudios de Traducción (como los denominó James Holmes en 1972) se alojaron en los departamentos de Lingüística y en otras en los departamentos de Letras Modernas, no debería sorprender un intercambio más fluido de la traducción con otros campos del saber.

Fue justamente el momento bisagra en que la Traductología devino Estudios de Traducción cuando James Hayes (1975) realiza estudios que ponen en tensión nociones anteriores sobre el dilema de preservar la forma o el contenido en literariedades del alemán. Si bien Hayes subraya "la imposibilidad de establecer un único set de principios aplicables a toda traducción" (1975: 838), aún mantiene un enfoque en cierta medida prescriptivo tomando las sugerencias de Eugene Nida sobre la traducción de poesía clásica griega (Hayes, 1975: 842), aunque observa la naturaleza misma del texto como factor pivotal para delimitar la tarea de traducirlo (Hayes, 1975: 838). Asimismo, considera el efecto de la textualidad (o el público lector) como segunda variable y ubica en tercer lugar la importancia del traductor en el proceso (Hayes, 1975: 838), lo que pone en cuestión ideas novedosas como la subjetividad del agente mediador de la traducción, que se convierte así en agente subjetivo.

Como se indicó, su postura, que se debate entre el estudio descriptivo de casos y la prescriptibilidad, subraya la equivalencia de contenido y forma (Hayes, 1975: 839) como objetivo principal de la traducción, agregando a su vez que es la cadencia de un texto con características poéticas lo que ofrece al traductor la mayor dificultad (Hayes, 1975: 839). Su análisis resulta interesante porque abre el camino de los Estudios de Traducción a un enfoque descriptivo que en la década siguiente habilitaría el giro cultural de la traducción propiciado por Susan Bassnett y André Lefevere (Bassnett, 2014: 30). Hayes (1975) sostiene que "si el traductor ha de transmitirlos [la forma y el contenido] con éxito, debe comprender con claridad su función en la trama como una unidad artística" (843). Por tanto, determina que una traducción confiable es aquella que transmite toda la información en términos rítmicos además de ofrecer el efecto del original (843). Hayes concluye que es la labor del crítico contribuir a que el traductor comprenda la naturaleza de los problemas a resolver más que adherirse con rigidez a cualquier solución preconcebida (848).

El estudio de Hayes resulta de particular interés por hallarse en la encrucijada de las ideas prescriptivas pasadas y los enfoques descriptivos en ciernes. Asimismo, plantea un interrogante que aún hoy posee relevancia: ¿qué sucede cuando la crítica no se ocupa de textualidades como la literatura infantil? La literatura infantil representa un área de poca exploración en los estudios literarios en general y más aún en los estudios de traducción.

Nitsa Ben-Ari (1992) fue una de las primeras estudiosas de la traducción de literatura infantil en comenzar a zanjar el vacío en el análisis de estas textualidades. Ben-Ari sostiene que existen ciertos principios relativos a la traducción de literariedades en donde las consideraciones de aceptabilidad priman siempre por sobre las consideraciones de adecuación (es decir la reconstrucción óptima del texto de 
origen) (Ben-Ari, 1992: 221), lo que implica que, más allá del nivel de canonicidad de un texto de origen (TO), una traducción debe ser aceptable en el sistema de la lengua de llegada. En este contexto, Ben-Ari agrega que la literatura infantil es por definición no canónica, y por tanto se ciñe con mayor rigidez a las normas que dominan la literatura infantil (1992: 222). Así, en la traducción de literatura infantil, Ben-Ari indica que hay normas operacionales cuya aplicación propende a hacer que el texto sea aceptable para los lectores en la lengua de llegada. Algunas de estas normas incluyen "normas de cancelación de repetición, adición y omisión [...] y diversas normas de conversión" (Ben-Ari, 1992: 223). Ben-Ari sostiene que la repetición no contribuye a que los lectores enriquezcan su vocabulario y por lo tanto recomienda la cancelación de repetición en los textos infantiles y fomenta la utilización de sinonimia. En relación con la omisión, Ben-Ari propone la eliminación de secciones completas según el modelo de llegada. Se puede hacer uso libre de adiciones, en especial con propósitos pedagógicos, incluyendo elementos de amplificación tales como conectores pragmáticos ("bueno", "en fin", etc.) o expresiones idiomáticas, y "elementos de explicación" para clarificar expresiones con el fin de facilitar la comprensión. Por último, menciona el uso de realia o "conversiones culturales" por medio de las que los nombres de comidas, bebidas o personas se traducen mediante equivalentes culturales (Ben-Ari, 1992: 277).

Shavit (1981) también se dedicó a estudiar las características específicas de la literatura infantil traducida del alemán al hebreo revisando variables históricas y entendiendo la fuerza canónica de ciertos libros dentro del catálogo del sistema. Shavit sostiene que "los traductores de literatura infantil se pueden permitir grandes libertades con respecto al texto como resultado de la posición periférica que ocupa la literatura infantil en el polisistema" (1981: 171) y por ello pueden manipular el texto de diversas formas mientras consideren dos principios sobre los que se basa generalmente la traducción de textos infantiles: la adaptación del texto para que sea apropiado y útil para los niños según las normas establecidas en una determinada sociedad, y la adaptación del texto al nivel de comprensión y las habilidades de lectura de un niño (Shavit, 1981: 171).

Sin embargo, ya en el siglo XXI, la misma Shavit expresa descontento sobre el estado de la investigación académica de esta literatura y afirma que "en el mundo académico de hoy, la investigación en literatura infantil no está legitimada, no es respetada y, si es tolerada, se percibe como un campo de estudio periférico e insignificante" (Shavit, 2003: 32). De Queiroga y Fernandes (2016) afirman que debido a la idea establecida de que la lectura de libros para niños requiere habilidades cognitivas simples y que "el rigor estético de los libros para niños se considera inferior en comparación con la literatura para adultos" (65), sumando al hecho de que cuestiones como la literariedad se consideran irrelevantes en textos cuyo propósito parece ser sólo la enseñanza, la literatura infantil se percibe como un "género simple [que] de inmediato se clasifica como noliteratura, nocanónico y nooficial" (65). En su caracterización del género, los autores sostienen que, en 
comparación con la traducción de literatura para adultos, "la literatura infantil puede ofrecer desafíos complejos que requieren mayor atención de parte del traductor en lo relativo a cuestiones teóricas y metodológicas" (de Queiroga y Fernandes, 2016: 70). Aspectos estilísticos, ideológicos y pedagógicos problematizan la traducción del género, sin obviar cuestiones atenientes al mercado editorial y los sistemas literarios de la cultura de llegada, que ponen en tensión la producción de traducciones de estas textualidades.

La teoría de polisistemas, desarrollada en los años 70 principalmente por Itamar Even-Zohar (2000), propone que la literatura es un subsistema de un sistema ideológico, socioeconómico e histórico que es dinámico y heterogéneo. Así, las obras literarias canónicas, que pertenecen al centro del sistema, y las obras no canónicas, que se ubican en su periferia, tienden a circular entre estos espacios y afectan el repertorio, entendido este como el conjunto de leyes y elementos que gobiernan la producción de obras literarias que se consumen en una determinada cultura (EvenZohar, 2000: 196-197).

La actividad traductora adquiere prominencia a través de esta propuesta que estudia la función de la literatura traducida y la considera como un sistema literario en sí mismo que se suma al sistema literario existente en una cultura de llegada. Según Even-Zohar (2000), las condiciones que deben darse para que obras innovadoras ingresen al sistema literario de llegada son que una literatura sea joven y esté en proceso de establecerse, que una literatura sea periférica o débil, o que haya momentos de inflexión, crisis o vacíos literarios en una literatura (197).

El presente artículo parte de la hipótesis de que la traducción de literatura infantil requiere de cierto grado de manipulación del texto de origen mediante distintas estrategias de traducción para que el producto terminado sea un texto que, al igual que ocurre con la literatura para adultos, se adapte a los modelos de la literatura de llegada. Nuestros objetivos persiguen el estudio de tres obras de traductores diferentes de la serie de Dr. Seuss a lo largo de un período de 40 años.

\section{John Hersey y sus habichuelas}

En mayo de 1954, la revista $L I F E$ dedicó una extensa nota a analizar el fracaso en la formación inicial de los niños estadounidenses. El artículo cuestionaba si los niños estaban aprendiendo a usar bien el lenguaje (Hersey, 1954: 136) basando sus observaciones en el informe de un comité escolar del distrito de Fairfield que señalaba el bajo rendimiento académico de los niños de primer grado. John Hersey, su autor, hallaba la causa de este problema en un enfoque que consideraba erróneo en la formación infantil, "del principio sólido de que un niño feliz se predispone con mayor facilidad a aprender que uno infeliz, puede surgir la conclusión equivocada de que la escuela se convierta en algo así como puro entretenimiento" (Hersey, 1954: 137). Ya en 1954 Hersey detectaba que la lectura debía competir 
por la atención de los niños contra medios "más entretenidos" (137) como la televisión, el cine o las historietas. En tal sentido, el autor criticaba los materiales de estudio que ofrecían "ilustraciones insípidas" de niños "anormalmente corteses" e "inusualmente limpios" (137). El artículo de Hersey concluía con la necesidad de que los padres apoyaran el proceso educativo de sus hijos: "Estas cuestiones van dirigidas a cada padre de este país, ya que los maestros no pueden responderlas solos. Al abordarlas, las escuelas necesitan del apoyo de una ciudadanía informada. ¿Quién puede resentir este apoyo? Después de todo, es para el beneficio de nuestros niños" (Hersey, 1954: 150). Esta fue la semilla que llevó a que Random House le comisionara a Theodor Seuss Geisel, mejor conocido como Dr. Seuss, redactar un libro para niños que ofreciera un vocabulario de 200 palabras nuevas. Lo que Dr. Seuss desconocía en ese momento es que la publicación de The Cat in the Hat en 1957 sería un punto de inflexión en su carrera como autor e ilustrador de libros infantiles.

\section{Cuando el gato no está, los traductores juegan}

The Cat in the Hat fue traducido al castellano en 1967, diez años después de su publicación en inglés, en 1957, por Carlos Rivera para Random House y publicado en una edición bilingüe titulada The Cat in the Hat/El Gato Ensombrerado. En el cuento, un gato vestido con un sombrero visita a dos niños un día gris y lluvioso para entretenerlos con juegos inusuales.

La estrategia más utilizada por Dr. Seuss es la rima, uno de los aspectos centrales de la literatura infantil que, junto con las onomatopeyas y los juegos de palabras, entre otros recursos que se destacan aún más en una lectura en voz alta, requieren "un alto nivel de creatividad por parte del traductor" (O'Sullivan, 2013: 455):

The sun did not shine

It was too cold to play

So we sat in the house

All that cold, cold, wet day

(Geisel, 1995: 3).
El sol no brillaba

Estaba demasiado mojado para jugar

Así es que nos sentamos adentro de la casa

todo aquel frío, frío día mojado (3).

A excepción del cambio de "frío" a "mojado" en el segundo verso, que en lugar de leerse "Estaba demasiado frío para jugar" se lee "Estaba demasiado mojado para jugar", Rivera ha optado por una versión que traslada de manera lineal el contenido del TO y elimina su rima, su patrón rítmico y su métrica. Basándonos en la idea de Shavit de que la traducción de literatura infantil se ajusta a los modelos existentes de la cultura receptora (Shavit, 1981: 172), la versión de Rivera resulta cuestionable si consideramos que tanto en España como en Latinoamérica la rima es un elemento clave en los modelos de literatura infantil. 
Dr. Seuss crea la rima mediante el uso de preposiciones diferentes (about y out) y también mediante el recurso de repetición, que le agrega cadencia al texto y así propende al aprendizaje de vocablos y expresiones nuevas de manera lúdica. El efecto resultante es un cúmulo de palabras que riman y que pueden recordarse con facilidad:

\author{
He should not be here \\ He should not be about \\ He should not be here \\ When your mother is out! \\ (Geisel, 1995: 13)
}

\author{
El [sic] no debe estar aquí \\ El [sic] no debe andar acá y allá \\ ¡El [sic] no debe estar aquí \\ cuando la mamá de Uds. está fuera!
}

(13)

Rivera no transfiere la estrategia de repetición de Dr. Seuss sino que produce una traducción que refleja las nociones de cancelación de la repetición y sinonimia que propone Ben-Ari (1992). En este sentido, Rivera escoge traducir el infinitivo be como "estar" en el primer y tercer verso y reemplaza be por "andar" en el segundo. Como resultado, se evidencia una pérdida de la anáfora, del paralelismo y de la cadencia, elementos provistos en el TO para llamar la atención de los jóvenes lectores. Asimismo, el traductor (o lo que es más posible, la casa editora) comete el error tipográfico de quitarle la tilde al pronombre "Él" y más adelante no transmite el sentido del verbo modal negativo should not, que expresa la conveniencia de que algo no suceda y no una prohibición de que algo suceda como lo denota el verbo modal negativo "no debe" empleado en la traducción.

A pesar de que Rivera rompe el patrón rítmico del TO además de que parece olvidar su propósito pedagógico, su traducción ofrece giros que resultan interesantes tales como los momentos en que demuestra coherencia en su recreación del argumento, cuando opta por las formas diminutivas de "nada" y "pizca", que resultan quizás más atractivas para los niños por su aspecto lúdico:
We did nothing at all (4)
No hicimos nadita (4)
Not one little bit (5) ni una pizquita (5)

Sin olvidar todo lo que han avanzado los estudios de traducción desde las épocas de Rivera, a partir del análisis de estos ejemplos podría afirmarse que, al dar preferencia al contenido sobre la forma, Rivera no parece haber considerado el propósito estético y pedagógico de aprendizaje léxico de la literatura infantil tal y como los diseñó Theodor Seuss Geisel: sus estrategias de traducción borran la cadencia del TO y las posibilidades de aprendizaje mediante la repetición y el juego. En este sentido, esta versión no se ciñe al principio propuesto por Shavit (1981) de ajustar el texto para hacerlo apropiado y útil para el niño, basado "en la noción de que la literatura infantil es una herramienta para la educación" (Shavit, 1981: 172). 


\section{Huevos verdes, ¿traductora verde?}

Publicado en 1960 por Random House, el decimoctavo libro de Dr. Seuss, Green Eggs and Ham, gira en torno a un ser extraño llamado Sam-I-Am, que le insiste a un monstruo gruñón que pruebe un plato nuevo. Este personaje por fin accede y se da cuenta de que el plato no sabe mal. Este libro fue traducido al castellano como Huevos verdes con jamón por Aída E. Marcuse y publicado por Lectorum en 1992, 25 años después de The Cat in the Hat.

$\mathrm{Al}$ igual que otras obras de Dr. Seuss, la rima desempeña un papel fundamental en el desarrollo de la trama y en sus diálogos:

I do not like them,

Sam-I-Am

I do not like

green eggs and ham. (Geisel,

1960a: 12)
No, no me gustan nada, Juan Ramón.

No, no me gustan nada

los huevos verdes con jamón (Geisel, 1992: 12)

Marcuse aplica la técnica que Ben-Ari (1992) luego denominará técnica de conversión de realia, es decir conversiones culturales necesarias cuando las normas de la cultura de origen por algún motivo no resultan deseables en la cultura de llegada (227). Así, traduce el nombre de Sam como Juan, y luego como Juan Ramón: la distinción entre "I Am Sam" y "Sam-I-Am" (cuando el personaje sostiene distintos carteles con su nombre) se compensa en castellano con la distinción entre "Yo soy Juan" y "Yo soy Juan Ramón" respectivamente. Es importante notar que el número de letras de las traducciones de Marcuse se condicen con el tamaño de los carteles de los dibujos. La elección del segundo nombre, Ramón, es adecuada si consideramos que se trata de un nombre muy común en el mundo hispano y que rima con la palabra jamón.

Siguiendo el fin pedagógico de la obra, en otro pasaje, Dr. Seuss incluye tres adverbios de lugar diferentes: here, there, anywhere. Este último, presenta un desafío para la traductora:

I would not like them here or there.

I would not like them anywhere.

(Geisel, 1960a: 16)
No, no me gustarían, no los quiero aquí ni allá.

No, no me gustarían aquí, allá o más allá.

(Geisel, 1992: 16)

La estrategia de traducción empleada por Marcuse para el adverbio anywhere, desglosado en "aquí, allá o más allá", es la que Ben-Ari (1992) denomina "elementos de explicación", que son maneras de allanar un concepto que resulta enigmático ya sea por una política consciente al respecto o por otras razones (225). Si bien los 
adverbios de lugar en la traducción son dos, en lugar de tres como en el TO, Marcuse logra mantener la rima mediante el recurso de repetición ("aquí ni allá" / "aquí, allá o más allá), ausente en el TO.

En otros casos, la rima no está dada por la repetición sino por el uso de dos palabras semánticamente diferentes (en este caso house y mouse):

Would you like them

in a house

Would you like them

with a mouse? (Geisel, 1960a: 18)
¿Te gustarían

en un caserón?

¿Te gustarían

con un ratón? (Geisel, 1992: 18).

Como se observa, Marcuse vuelve a mantener la rima y, al mismo tiempo, también logra mantener el contenido. Así, si bien existe una diferencia semántica, y connotativa, entre house y "caserón", se preserva la naturaleza del significante, y por tanto se mantiene el equilibrio entre contenido y forma.

En otras instancias, la rima vuelve a estar dada por dos palabras diferentes, pero la estrategia de traducción es distinta:

$\begin{array}{ll}\text { Would you eat them } & \text { ¿Los comerías } \\ \text { in a box? } & \text { en un cajón } \\ \text { Would you eat them } & \text { con un zorro } \\ \text { with a fox? (Geisel, 1960a: 22) } & \text { en un rincón? (Geisel, 1992: 22) }\end{array}$

A los fines de mantener la rima, Marcuse se valió de la norma de omisión de BenAri (1992) como así también de la norma de adición: la estructura "Would you eat them" se repite dos veces en el TO, mientras que en la traducción (" ¿Los comerías [...]?") aparece solo una, y además se agrega contenido (“en un rincón”).

Como se observa, Marcuse ha utilizado estrategias para equilibrar el contenido y la forma del TO, lo que da como resultado una versión en castellano que ha preservado el atractivo del texto para un público infantil: mediante el empleo de normas como la adición y la omisión, la traducción responde a los dos principios propuestos por Shavit (1981) de que el texto sea apropiado y útil (cumple un fin pedagógico) y se adapte al nivel de comprensión y las habilidades de lectura de un niño.

\section{Espejito, espejito, ¿quién es la más bella del reino?}

One Fish, Two Fish, Red Fish, Blue Fish es el decimonoveno libro de Dr. Seuss y fue publicado en 1960 por la editorial Random House. La historia hace que el lector recorra las actividades de un grupo particular de animales. Fue publicado en castellano en 2006 por Lectorum con el título de Un pez, dos peces, pez rojo, pez azul en traducción de Yanitzia Canetti. 
Tras mostrar a los cuatro peces que dan nombre al título del cuento, Dr. Seuss presenta nuevos personajes empleando el mismo sustantivo cuatro veces, adjetivado dos veces con colores (black y blue) y dos veces con antónimos (old y new) y la rima está dada por la repetición de fish:

$\begin{array}{ll}\text { Black fish } & \text { Pez negro } \\ \text { blue fish } & \text { pez azulito } \\ \text { old fish } & \text { pez anciano } \\ \text { new fish (Geisel, 1960b: 4) } & \text { pez bebito (Geisel, 2006: 8) }\end{array}$

Canetti ha favorecido la rima y la musicalidad en su traducción y al mismo tiempo ha logrado trasladar el contenido del TO mediante la elección de equivalentes no literales de algunos de los adjetivos. Esto, asimismo, ha creado una personificación del pez que estaba ausente en el TO: en lugar de haber un pez "nuevo" y un pez "viejo", hay uno "anciano" y otro "bebito".

Asimismo, en otra sección, la rima está dada por palabras distintas (car y are) y no por repetición como en el ejemplo anterior:

This one has a little car

Say! What a lot

of fish there are. (Geisel, 1960b: 5)
Y este corre a todo dar

Va tan rápido en su auto

¡que se va a estrellar! (Geisel, 2006: 9)

En este caso la traductora opta por preservar la forma y dejar de lado el contenido, aunque mantiene la trama en torno a un automóvil. Al igual que Marcuse, una de las estrategias de Canetti es aprovechar las desinencias "-ar", "-er" e "-ir" de los verbos en infinitivo en español, que representan infinitas opciones para la rima: aquí la rima está lograda mediante los infinitivos "dar" y "estrellar" y, si bien el pez tiene un automóvil como en el TO (una situación referida a través de la imagen de un pez en un coche), la traductora advierte que lo conduce demasiado rápido, lo que aporta una enseñanza que estaba ausente en el TO, siguiendo las ideas de Ben-Ari (1992) relativas al uso libre de adiciones con propósitos pedagógicos como en el caso de esta amplificación.

\section{Y colorín, colorado...}

Las discusiones sobre literatura infantil allanan el camino subestimado y relegado, y por lo tanto casi inexplorado, de la investigación académica de estas textualidades y así posibilitan los dos cambios que según Shavit (2003) deben darse para que se revierta el estatus de inferioridad de este género: la literatura infantil debe ser aceptada como un campo legítimo de investigación académica y debe ser considerada como un sistema autónomo de los sistemas literarios que a su vez forman el 
conglomerado de sistemas culturales (33). Asimismo, las discusiones sobre la traducción de literatura infantil revelan las normas traslativas que ponen en evidencia las restricciones que se le imponen a un texto que ingresa en un determinado sistema de literatura infantil. De las traducciones analizadas, las más efectivas en cuanto al propósito pedagógico analizado por Ben-Ari (1992) y a los principios propuestos por Shavit (1981) de adaptar el texto de modo que sea apropiado y útil, y para que se adecue a las habilidades de lectura de un niño, son aquellas en las que se preservaron la rima, la cadencia y la repetición sin que esto vaya en detrimento del contenido. Este equilibrio se encuentra en algún punto medio entre lo que de Queiroga y Fernandes (2016) observan como un riesgo inherente a las elecciones del traductor entre una traducción literal y una traducción libre: "una proximidad excesiva al texto de origen (literalidad) puede generar falta de vitalidad o dificultar la lectura (falta de legibilidad), mientras que una versión adaptada puede eliminar elementos considerados esenciales que son fruto de la intención del autor" (69). El abordaje palabra por palabra de Rivera permite la transferencia de la información, pero no de la poesía de Dr. Seuss, lo que da como resultado un producto cuestionable en cuanto a lo que Hayes (1975) determina como una traducción confiable: aquella que transmite toda la información en términos rítmicos además de ofrecer el efecto del original (843). Marcuse y Canetti parecen haber producido traducciones exitosas en cuanto a crear un texto que sea "placentero y motivador y así aliente a los lectores infantojuveniles a seguir leyendo" (de Queiroga y Fernandes, 2016: 75) mediante el uso de estrategias y técnicas similares: la adición de rimas ausentes en el TO, el uso de elementos lingüísticos disponibles en español y no en inglés, y las conversiones de elementos de explicación y realia que propone Ben-Ari (1992). Marcuse y Canetti han repetido ciertos patrones y aun así han introducido nuevos elementos léxicos en sus traducciones. La repetición, por tanto, parece una condición sine qua non para retratar la esencia real de los libros de Dr. Seuss en español. No es sólo una cuestión de forma, mediante la que se construye un patrón rítmico, sino también una cuestión de contenido: en Green Eggs and Ham, la repetición de palabras y acciones en SamI-Am cumplen la función de que los lectores tomen consciencia de la importancia de la coherencia y la perseverancia necesarias para lograr metas en la vida.

El comportamiento de la traducción de literatura infantil está en gran medida determinado por la posición de la literatura infantil en el polisistema literario (Shavit, 2006: 25). Las casas editoras reconocen la importancia institucional (a través de las políticas públicas fomentadas desde los estados nacionales y, en un nivel más inmediato, desde las escuelas) en la conformación del canon literario. La gerente editorial del Grupo Planeta, Adriana Fernández, sostiene que "Lo infantil tiene, por una lado [sic], la perspectiva literaria [...] que van sosteniendo o armando canon [...]. En esa lógica no sólo está la mirada de la crítica, sino la de la escuela que es quien, a su manera, también crea canon” (León, 2017). En sintonía, la editora María Fernanda Maquieira, coincide en resaltar el rol de la escuela como 
"agente fundamental para la formación de lectores [al igual que maestros y bibliotecarios y familias, todos comprometidos] con el tema de la lectura en la primera infancia" (León, 2017). Se observa, por tanto, que los participantes clave en la promoción de la lectura no han variado de manera considerable desde la publicación del artículo de Hersey a pesar del tiempo transcurrido y la distancia geográfica con Argentina, y que el consumo de literatura infantil está asociado a propósitos de instrucción, moral y educación, lo que demuestra una fuerte inclinación pedagógica de la literatura infantil (Lathey, 2010: 3).

En Argentina, como sostiene la directora de la editorial Comunicarte, Karina Fraccarolli (comunicación personal, 9 de junio de 2020), el sistema literario de literatura infantil de producción local es sólido y establecido, de modo que, más allá de la calidad de las traducciones, es lógico que los productos literarios importados encuentren resistencia para ingresar al sistema (Entrevista). Más bien, tienden a quedar circulando en la periferia hasta desaparecer, como fue el caso de estos libros de Dr. Seuss. Así, desde una mirada polisistémica, es muy probable que el fracaso desde el punto de vista comercial de los libros de Dr. Seuss en Argentina (ausentes de toda lista de libros mejores vendidos) se deba a que en el sistema literario argentino existe una tradición de larga data de autores prestigiosos cuyas obras literarias se destacan por la construcción de la rima, como por ejemplo María Elena Walsh. La autora abogaba en una entrevista de 1998 por trabajar el lenguaje de manera creativa: "Sólo me interesaba que los chicos se animaran a jugar con el lenguaje, a abordar la realidad de una manera creativa. Que gozaran de una estética de la música y de la letra" (Clarín). La aceptación de estas obras, por lo tanto, no sólo recae en la calidad de las traducciones sino en la sólida posición de la literatura nacional que el público infantil y adulto está acostumbrado a leer y aceptar. Si bien, siguiendo a Ben-Ari (1992) y Shavit (1981), y recuperando las ideas de Hayes (1975) sobre forma y contenido, algunas de las traducciones que analizamos fueron efectivas en cuanto a adaptarse al modelo de la literatura de llegada al mantener la rima y la intención pedagógica del TO, es quizás la impermeabilidad del sistema literario argentino que apoya institucionalmente las producciones vernáculas, la razón por la que la serie de Dr. Seuss no halló espacio efectivo en este país. Retomando las ideas de Even-Zohar (2000), quizás no estaban dadas las condiciones para que estas obras traducidas ingresaran al canon literario argentino, es decir, la literatura infantil no era joven ni estaba en proceso de establecerse, tampoco era periférica o débil, ni hubo en ese momento una crisis o un vacío literario en la literatura infantil argentina. Sin embargo, más allá de que estos textos traducidos sean un fracaso o un éxito comercial, el estudio de la traducción de literariedades infantiles, cuya producción (escritura, traducción, publicación, crítica, recomendación y venta) está en un todo en manos de los adultos hasta que el producto terminado llega al receptor (O'Sullivan, 2013: 456), enriquece el campo de los estudios de traducción de literatura infantil que, como vimos en este trabajo, aún tienen un estatus de inferioridad, y además puede 
abrir el camino para que traducciones futuras enriquezcan y complementen el repertorio literario argentino.

\section{Referencias bibliográficas}

BASSNETT, Susan. (2014). Translation. Londres: Routledge.

BEN-ARI, Nitsa. (1992). "Didactic and Pedagogic Tendencies in the Norms Dictating the Translation of Children's Literature: The Case of Postwar GermanHebrew Translations". Poetics Today, 13(1), Children's Literature, 221-230. https://doi.org/10.2307/1772799

Clarín. "A fondo. Maria Elena Walsh, escritora. 'En la Argentina la gente adulta tiene conductas infantiles"” (en línea). Disponible en https:/www.clarin.com/ opinion/argentina-gente-adulta-conductas-infantiles_0_H16tNZk8hg.html

DE QueIroga, Marcílio García, y Fernandes, Lincoln Paulo. (2016). "The Translation of Children's Literature". Cadernos de Tradução, 36(1), 62-78. https:// doi.org/10.5007/2175-7968.2016v36n1p62

Even-ZoHAR, Itamar. (2000). "The Position of Translated Literature within the Literary Polysystem”. En Lawrence Venuti (Comp.), The Translation Studies Reader. Londres: Routledge. 192-197.

GeIsel, Theodor Seuss. (1960a). Green Eggs and Ham. Nueva York: Random House.

GeISEL, Theodor Seuss. (1960b). One Fish, Two Fish, Red Fish, Blue Fish. Nueva York: Random House.

GeISEL, Theodor Seuss. (1992). Huevos verdes con jamón (Aída E. Marcuse, Trad.). Nueva York: Lectorum Publications.

GeISEL, Theodor Seuss. (1995). The Cat in The Hat: El Gato Ensombrerado. (Carlos Rivera, Trad.). Nueva York: Random House.

GeISEL, Theodor Seuss. (2006). Un pez, dos peces, pez rojo, pez azul. (Yanitzia Canetti, Trad.). Nueva York: Lectorum Publications.

HAYES, James A. (1975). "The Translator and the Form-content Dilemma in Literary Translation”. MLN, 90(6), 838-848. http://doi.org/10.2307/2907023

Hersey, John. (1954, mayo). "Why Do Students Bog Down on First R?". Life, 36(21). 136-150.

Holmes, James. (2000). "The Name and Nature of Translation Studies". En Lawrence Venuti (Comp.), The Translation Studies Reader. Londres: Routledge. 172-185.

LATHEY, Gillian. (2010). The Role of Translators in Children's Literature. Invisible Storytellers. Nueva York: Routledge.

LEÓN, Gonzalo. (2017, 5 de julio). "Los caminos de la literatura infantil y juvenil: ¿lo mejor está por venir?” (en línea). Infobae. Disponible en https://www.in- 
fobae.com/cultura/2017/07/05/los-caminos-de-la-literatura-infantil-y-juvenillo-mejor-esta-por-venir/

O’Sullivan, Emer. (2013). “Children's Literature and Translation studies”. En Carmen Millán y Francesca Bartrina (Eds.), The Routledge Handbook of Translation Studies. Nueva York: Routledge. 451-463.

SHAVIT, Zohar. (1981). "Translation of Children's Literature as a Function of Its Position in the Literary Polysystem". Poetics Today, 2(4), Translation Theory and Intercultural Relations, 171-179. http://doi.org/10.2307/1772495

SHAVIT, Zohar. (1992). "Literary Interference between German and Jewish-Hebrew Children's Literature during the Enlightenment: The Case of Campe". Poetics Today, 13(1), Children's Literature, 41-61. http://doi.org/10.2307/1772788

SHAVIT, Zohar. (2003). "Cheshire Puss, Would you tell me, please, which way I ought to go from here? Research of Children Literature: The State of Art. How Did We Get There; How should we proceed". En Ana I. Labra Cenitagoya; Esther Laso y León; y José Santiago Fernández Vázquez (Coords.), Realismo social y mundos imaginarios, una convivencia para el siglo XXI. Alcalá de Henares: Universidad de Alcalá, Servicio de Publicaciones. 30-41.

SHAVIT, Zohar. (2006). "Translation of Children's Literature". En Gillian Lathey (Ed.), The Translation of Children's Literature: A Reader. Clevedon: Multilingual Matters Ltd. 25-40. 